\title{
UV-Schutz kann zu Vitamin-D-Mangel führen
}

UV-Strahlen sind der wichtigste Risikofaktor für Schäden der Haut. Meiden Menschen die Sonne komplett, können sie nicht mehr genügend Vitamin D in der Haut synthetisieren. Hier sollte man entweder über die Ernährung vorsorgen, mit Vitamin D supplementieren oder, sofern möglich, für ein paar Minuten Sonnenlicht täglich sorgen.

Ü bermäßige UV-Strahlung schadet der Haut, doch Licht ist gleichzeitig essenziell für die Vitamin-DSynthese, die in unseren Breitengraden zu etwa 90\% mit Hilfe von UV-B-Strahlung in der Haut stattfindet. Vitamin-D-Mangel gilt als Risikofaktor oder verschlechtert die Prognose bei einer Reihe maligner Erkrankungen, Autoimmunerkrankungen, Infektionen und kardiovasulären Erkrankungen.

Als Reaktion auf steigende Raten von Hauterkrankung warnen Präventionskampagnen vor UV-Strahlung: Pilotstudien bei Patientenpopulationen, die wegen ihres bekannt hohen Risikos für UV-Schäden konsequenten Sonnenschutz betreiben (nach Nierentransplantation, Xeroderma pigmentosum), zeigen deutlich verminderte Vitamin-D-Spiegel.

Wie viel Vitamin D wir eigentlich brauchen, ist unklar. US-Empfehlungen von 1989 (Recommended Dietary Allowance, RDA) mit 200 IU gelten als veraltet, auch aktuelle Empfehlung von 600 IU (Daily Reference Intake, DRI) scheinen noch zu gering, Man geht aktuell von einem Bedarf zwischen 4.000 und 10.000 IU täglich aus, toxische Nebenwirkungen werden ab 40.000 IU vermutet. In Breitengraden wie in Boston oder Rom werden an einem Sommertag in Badebekleidung bei kurzer Sonnenexposition mit der minimalen ErythemDosis von fünf Minuten (Hauttyp 2) 10.000 IU Vitamin D synthetisiert. Sind nur Gesicht, Arme und Hände exponiert $(<18 \%$ Oberfläche) reichen nach Schätzungen zwischen Frühjahr und Herbst ein Drittel bis die Hälfte der minimalen Erythem-Dosis (von fünf Minuten) an zwei bis drei Tagen pro Woche für den Grundbedarf des Vitamins aus.

Fazit: Mit der täglichen Gabe von 1.000-2.000 IU Vitamin D kann ein Mangel bei Risikopopulationen mit geringer Sonnenexposition kompensiert werden. Dermatologen sollten an Vitamin-D-Mangel denken, wenn sie gefährdeten Patienten zu starkem Sonnenschutz raten.

$\mathrm{mf}$

Reichrath J. Skin cancer prevention and UV-protection: how to avoid vitamin D-deficiency? Br J Dermatol. 2009;161 Suppl 3:54-6o. 